\title{
Tactile selective attention and temporal masking
}

\author{
JAMES C. CRAIG \\ Indiana University, Bloomington, Indiana \\ and \\ PAUL M. EVANS \\ Willamette University, Salem, Oregon
}

\begin{abstract}
The presentation of a nontarget stimulus to one fingerpad interferes with the identification of a target stimulus presented to a second fingerpad. This interference has been attributed to a failure of selective attention and, more specifically, to the nontarget's eliciting a competing response. In the present study, the temporal interval between the target and nontarget was varied to determine the extent to which a nontarget primes a competing response. The results showed more interference when the nontarget was presented after the target than when it was presented before the target. Although still consistent with a response-competition explanation, this result offered no support for a priming explanation. The function relating the amount of interference to the temporal separation between the target and nontarget was similar to the functions obtained in studies of temporal masking, and this prompted a second experiment in which temporal masking was examined. These results, obtained with stimuli presented to the same fingerpad, indicate that response competition may be a major factor in temporal masking and that similar processes are involved in temporal masking and selective attention.
\end{abstract}

The ability of subjects to identify a target pattern presented to one location on the skin and to ignore a nontarget pattern presented to another location on the skin has been examined in several recent studies (Evans \& Craig, 1991, 1992; Evans, Craig, \& Rinker, 1992). Since the results of these studies have shown that a nontarget interferes with the identification of a target, it has been concluded that subjects have difficulty in restricting their attention to a single site on the skin. Additional conclusions drawn from these studies were (1) that the pattern presented to a nontarget site is processed to the point of activating a response, and (2) that the majority of the interference effect results from the nontarget's activating a response that differs from, and competes with, the target response.

The latter conclusion, that interference occurs as a result of response competition, is based on results from what has been referred to as a "4-to-2" paradigm. In this paradigm, also used in studies of selective attention with visual stimuli (Eriksen \& Schultz, 1979), a subject is presented with one of four patterns at a target site. Of the four patterns, two are associated with one response and two are associated with a second response. One of the four patterns is also presented to the nontarget site. The pairing of target and nontarget patterns creates three types

\footnotetext{
This research was supported by Grant DC-00095 from the National Institutes of Health and was conducted while the second author was a visiting scholar in the Institute for the Study of Human Capabilities at Indiana University. The authors thank Roger P. Rhodes for his assistance in conducting these experiments. Reprint requests should be addressed to J. C. Craig, Department of Psychology, Indiana University, Bloomington, IN 47405 (e-mail: craigj@ucs.indiana.edu)
}

of trials. In one type of trial, the target and nontarget are physically identical (stimulus-compatible trials [SC]). In the second type of trial, the target and nontarget are physically different but associated with the same response (response-compatible trials $[\mathrm{RC}]$ ). In the third type of trial, the target and nontarget stimuli are physically different and associated with different responses (response-incompatible trials [RI]).

The primary reason for using the 4-to-2 paradigm is to measure the amount of interference produced by a nontarget that might be attributed to a process occurring at a relatively early stage of pattern processing, such as masking. If a nontarget interferes with a target at a relatively early stage of processing, then, it has been argued, a nontarget that is physically different from a target should interfere with the identification of the target. The interference should occur regardless of the response with which it is associated. On the other hand, if a nontarget interferes with a target at a relatively late stage of processing by evoking a competing response, then the nontarget that is physically different from the target but evokes the same response should produce little interference. The tactile results, paralleling those reported by C. W. Eriksen and his colleagues with visual stimuli (Eriksen \& Schultz, 1979; Eriksen, O'Hara, \& Eriksen, 1982), supported the latter view. As long as the nontarget was associated with the same response category as the target, little interference was observed. When the nontarget was associated with the opposite response category, considerable interference was observed, in terms of both reduced accuracy and increased reaction times (Evans \& Craig, 1992; Evans et al., 1992). These results were interpreted to suggest that little masking occurs 
between target and nontarget stimuli presented to separate sites. The major portion of the observed interference is due to the nontarget's evoking a response that competes with the target response.

In a selective-attention paradigm in which the target and nontarget stimuli are presented to separate sites, subjects' failure to correctly identify the target may be due, in part, to the fact that cues associated with spatial location are insufficient to keep the two stimuli separated. It may be as if the target and nontarget patterns were presented to the same location. The presentation of two patterns at the same location is the paradigm generally used in temporal-masking studies. Indeed, the major difference between a temporal-masking paradigm and a selective-attention paradigm is in the location of the nontarget stimulus, the nontarget being presented either to the same site as the target or to a diffferent site. In many studies of temporal masking, the major cue available to the subject to distinguish target from nontarget (masker) is the temporal position of the target. Does the target come before or after the nontarget? Typical results from temporal-masking studies show more backward than forward masking, that is, more interference when the masker is presented after the target pattern (Craig, 1982, 1985). This finding runs contrary to views of the response-competition effect in selective attention, namely, that the target primes a competing response (Eriksen \& Schultz, 1979; Evans \& Craig, 1992). Priming implies that a nontarget would interfere only when it was presented before (or simultaneously with) the target rather than after the target. One would not expect a trailing nontarget stimulus to prime a competing response more effectively than a leading nontarget stimulus. In support of this view, Eriksen and Schultz (1979), in a study of visual selective attention, found little interference when a nontarget trailed a target stimulus; however, in a recent study of selective attention with tactile stimuli, significant interference was found with a nontarget stimulus trailing the target stimulus. In one condition, there appeared to be even greater interference with a trailing nontarget than with a leading nontarget (Rinker \& Craig, 1994).

The present study was undertaken to examine the temporal course of interference in a selective-attention paradigm and to see if priming was an adequate explanation of the response competition effect (Experiment 1). The results from these measurements led us to consider similarities between selective-attention and temporal-masking functions (Experiment 2).

\section{EXPERIMENT 1}

The time course of selective attention was examined using nontargets that preceded or followed a target. The target site was the little finger; the nontarget site was the ring finger. In a control condition, the target and nontarget sites were switched. This control condition was included to examine the possibility that processing at one site on the skin might be substantially slower than pro- cessing at another site. If there was such a difference in processing time, it could lead to errors in interpreting the time course of selective attention.

\section{Method}

Subjects. The subjects were undergraduate students at Indiana University. Seven subjects were tested. All were right-handed. All of the subjects had participated in a number of related experiments in the laboratory and were paid for their participation. In the control experiment, 4 subjects were tested. All 4 had participated in the main experiment.

Apparatus. Details of the apparatus may be found in Craig (1980). Tactile stimuli were presented to subjects' fingerpads using two tactile arrays from an Optacon, a reading machine for the blind. Each array consists of 144 stimulators, 6 columns by 24 rows. The arrays fit against the subject's fingerpads. By activating the stimulators in the arrays sequentially, the perception of movement across the fingerpad was created (see Evans \& Craig, 1991, 1992). A computer controlled the tactile displays, the presentation routine, and a visual display used to present instructions and feedback. The computer was also used to collect subjects' responses, including response latencies.

Stimuli. The stimuli consisted of vertical and horizontal bar-like patterns that appeared to move across the fingerpad in one of four directions, left, up, right, or down. The total duration of each stimulus was $52 \mathrm{msec}$. Further details about the generation of the stimuli can be found in Evans and Craig (1991, 1992).

Procedure. The subjects were tested individually. Each subject sat with his/her left little finger resting on one display and his/her left ring finger resting on the second display. The subjects were told that they would be presented with two tactile stimuli, one to each finger, and that the stimuli would appear to be moving to the left, right, up, or down. They were instructed that their task was to categorize the direction of movement on the little finger (target site) and to ignore the stimulation on the ring finger (nontarget site). For 3 of the subjects, if the target stimulus moved to the left or up, they were to respond "one." If the target moved to the right or down, they were to respond "two" (Evans \& Craig, 1992, Figure 1). For the remaining 4 subjects, the stimulus pairing was changed. If the target stimulus moved to the left or down, they were to respond "one," and if it moved to the right or up, they were

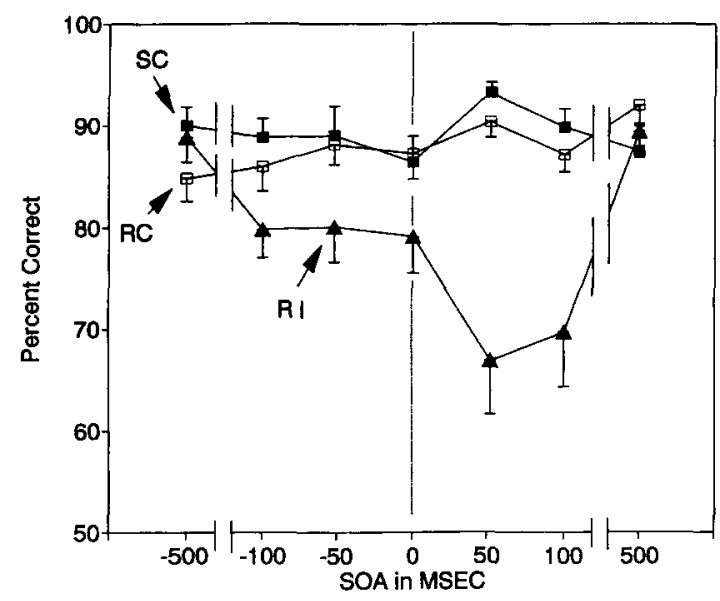

Figure 1. Percent correct identification for the three trial typesstimulus compatible (SC), response compatible (RC), and response incompatible (RI) - as a function of SOA. The target site was the little finger; the nontarget site was the ring finger. Error bars represent 1 SEM. 
to respond "two." All subjects responded by pressing the two response keys with the index and middle fingers of their right hands. Accuracy was stressed, although the subjects were aware that their responses were being timed.

There were three trial types. The selection and pairing of target and nontarget stimuli were done randomly on a trial-by-trial basis. Thus, on approximately one-fourth of the trials, the target and nontarget stimuli were identical and, of course, were associated with the same response (stimulus-compatible, SC, trials). On another fourth of the trials, the target and nontarget stimuli were different but were associated with the same response (response-compatible, $\mathrm{RC}$, trials). On the remaining half of the trials, the stimuli were different and associated with different responses (responseincompatible, RI, trials).

A subject initiated each trial by pressing either one of the two response keys. One second later, the stimulus sequence began. Following presentation of the target and nontarget stimuli, the subject indicated his/her response and received feedback via the visual display. A correct response was followed by the word "CORRECT" appearing on the visual display. An incorrect response was followed by the word "WRONG" on the display. The subject then initiated the next trial by pressing one of the response buttons. To prevent auditory cues from the tactile displays influencing their responses, the subjects wore earplugs and headphones through which white noise was presented.

Seven stimulus onset asynchronies (SOAs) between the target and nontarget were tested, $-500,-100,-52,0,+52,+100,+500$, where negative SOAs refer to trials in which the nontarget preceded the target and positive SOAs indicate the reverse. Trials were blocked by SOA. Each session consisted of eight blocks, of 50 trials each, including one block in which the target was presented by itself. Each session began with a single-pattern block of trials followed by the remaining seven blocks in random order. The subjects were tested for eight sessions.

A control condition tested whether the site of stimulation affected the function relating interference to SOA. For half of the sessions (seven blocks per session) in this condition, the target and nontarget sites were the same as they had been in the main experiment, little and ring fingers, respectively. For the other half of the sessions, they were switched, with the ring finger serving as the target site and the little finger as the nontarget site. Three SOAs, $-78,0$, and $+78 \mathrm{msec}$, were tested. The subjects were tested in six sessions, three with the little finger as target site followed by three with the ring finger as target site.

\section{Results and Discussion}

The results from the main experiment were first examined to see if changing the stimulus-response pairing had an effect on performance. The overall difference for the two groups of subjects was less than $3 \%$ correct, so the results were combined for subsequent analysis. Singlepattern performance in the main experiment was $93 \%$ correct. Performance in the presence of the nontarget varied as a function of the temporal separation between the target and nontarget. The results for the three trial types are shown in Figure 1. As in previous studies of selective attention, performance on SC and RC trials appears to remain unchanged as a function of SOA; performance on RI trials, however, did change as a function of SOA. It appears that greater interference is seen when the nontarget follows rather than leads the target. The results were subjected to a two-way, repeated measures analysis of variance (ANOVA), trial type $\times$ SOA, and the analysis supported these initial observations. The ANOVA revealed a significant main effect of SOA
$[F(6,36)=3.50, p<.01]$ and a significant main effect of trial type $[F(2,12)=14.05, p<.001]$. There was an interaction between SOA and trial type $[F(12,72)=9.59$, $p<.001]$. We were particularly concerned with performance on the RI trials at briefer SOAs. Specifically, we wanted to determine if performance on RI trials was significantly lower when the nontarget followed the target than when it preceded the target. Such was the case. A Tukey $H S D$ test showed that performance, as measured by changes in percentage correct, was significantly lower at $+52 \mathrm{msec}$ than at $-52 \mathrm{msec}(p<.05)$. Also, performance was significantly lower at $+52 \mathrm{msec}$ than at 0 $\operatorname{msec}(p<.05)$.

As in previous studies of tactile selective attention, the main measure of performance was accuracy, and the instructions to the subjects emphasized this. Reaction times were recorded, primarily to determine if the responsecompetition effect was due to a speed-accuracy tradeoff, but also to shed some light on the nature of the information that subjects have available at the time that they make their decisions. No speed-accuracy tradeoff was evident. Indeed, the opposite effect was observed. The ANOVA showed a main effect of SOA $[F(6,36)=$ $8.35, p<.001]$, a main effect of trial type $[F(2,12)=$ $16.23, p<.001]$, and a significant interaction between SOA and trial type $[F(12,72)=3.53, p<.001]$. As observed in previous studies (Evans \& Craig, 1991, 1992; Evans, Craig, \& Rinker, 1992), reaction times were longer for RI trials than for SC and RC trials. The reaction time on SC trials averaged across the five briefest SOAs was $428 \mathrm{msec}$. The comparable value for RC trials was $444 \mathrm{msec}$ and for RI trials, $456 \mathrm{msec}$. These results suggest that subjects have available representations of both the target and the nontarget at the time that they make their decisions. Longer reaction times are associated with choosing between representations and, on RI trials, between competing responses as well.

The change in the amount of interference as the SOA varies from -100 to $+100 \mathrm{msec}$ is not simply due to the skin's being insensitive to relatively small changes in the time between the onsets of the target and the nontarget. If the percentage correct when the nontarget was presented $52 \mathrm{msec}$ after the target was similar to that obtained when the nontarget was presented $52 \mathrm{msec}$ before the target or simultaneously with the target, one might simply attribute the results to temporal insensitivity or uncertainty. The results do not support such a conclusion. When the nontarget is delayed by $52 \mathrm{msec}$ relative to the target, there is a large drop in percentage correct. Clearly, the skin is very sensitive to temporal changes on the order of approximately $50 \mathrm{msec}$, and in a direction opposite to that predicted by a priming explanation. A priming explanation of the response-competition effect with tactile stimuli would predict more "forward" than "backward" interference.

As noted before, we also considered the possibility that there might be substantial differences in processing time between the target and nontarget sites (Eriksen \& Schultz, 1979). If the pattern presented to the ring fin- 
ger (the nontarget site) were processed more rapidly than the pattern presented to the little finger (target site), then, for the two stimuli to be perceived as simultaneous, the nontarget would have to trail the target. Under such circumstances, targets presented to the ring finger would enjoy a substantial temporal advantage. If priming were the explanation for the results shown in Figure 1, then nontargets presented to the little finger should produce interference only when the nontarget leads the target by a substantial amount and there should be no interference seen with a trailing nontarget. In other words, performance should improve as the nontarget moves to a trailing position. The results from the control experiment testing this possibility are shown in Figure 2.

The results show that the RI functions are very similar in the two conditions. There is no evidence for any differences in processing time at the two sites, particularly any difference that would account for there being greater interference produced by trailing nontargets. Single-pattern performance was $96 \%$ correct on the little finger and $94 \%$ correct on the ring finger. A two-way, repeated measures ANOVA with the little finger as target site (ring finger as nontarget site) showed a significant effect of SOA $[F(2,6)=14.89, p<.01]$, a significant effect of trial type $[F(2,6)=22.93, p<.01]$, and a significant interaction between SOA and trial type $[F(4,12)=15.02, p<.001]$. With the ring finger as target site (little finger as nontarget site), the ANOVA showed no effect of SOA $[F(2,6)=4.04, p>.05]$, a significant effect of trial type $[F(2,6)=75.26, p<.001]$, and a significant interaction between trial type and SOA $[F(4,12)=7.19, p<.01]$. These results also replicate the main effect shown in Figure 1, namely, that a trailing nontarget produces more interference than a leading nontarget and demonstrate that this effect is independent of the site of stimulation.

A priming explanation of the response-competition effect implies, by definition, that greater interference should be observed when the prime (nontarget) precedes the onset of a target. The results of Experiment 1 clearly show that significantly more interference occurs when a nontarget follows the onset of a target, and are thus inconsistent with a priming explanation. If priming does not explain the response-competition effect, then what causes the increase in the probability of subjects' selecting the nontarget when it follows rather than precedes the target? Studies of temporal masking with stationary patterns have generally found more backward than forward masking (Craig, 1976, 1982, 1985; Evans, 1987; Evans \& Craig, 1986). This difference has been attributed in part to the later-arriving pattern's being more strongly represented than the earlier pattern (Evans $\&$ Craig, 1986). In the context of selective attention, it would mean that subjects select the more strongly represented trailing nontarget. According to this view, raising the intensity ought to increase the amount of interference in a selective-attention task. Evans and Craig (1992) raised the amplitude of the nontarget to a level judged to be 2.5 times greater than that of the target. This
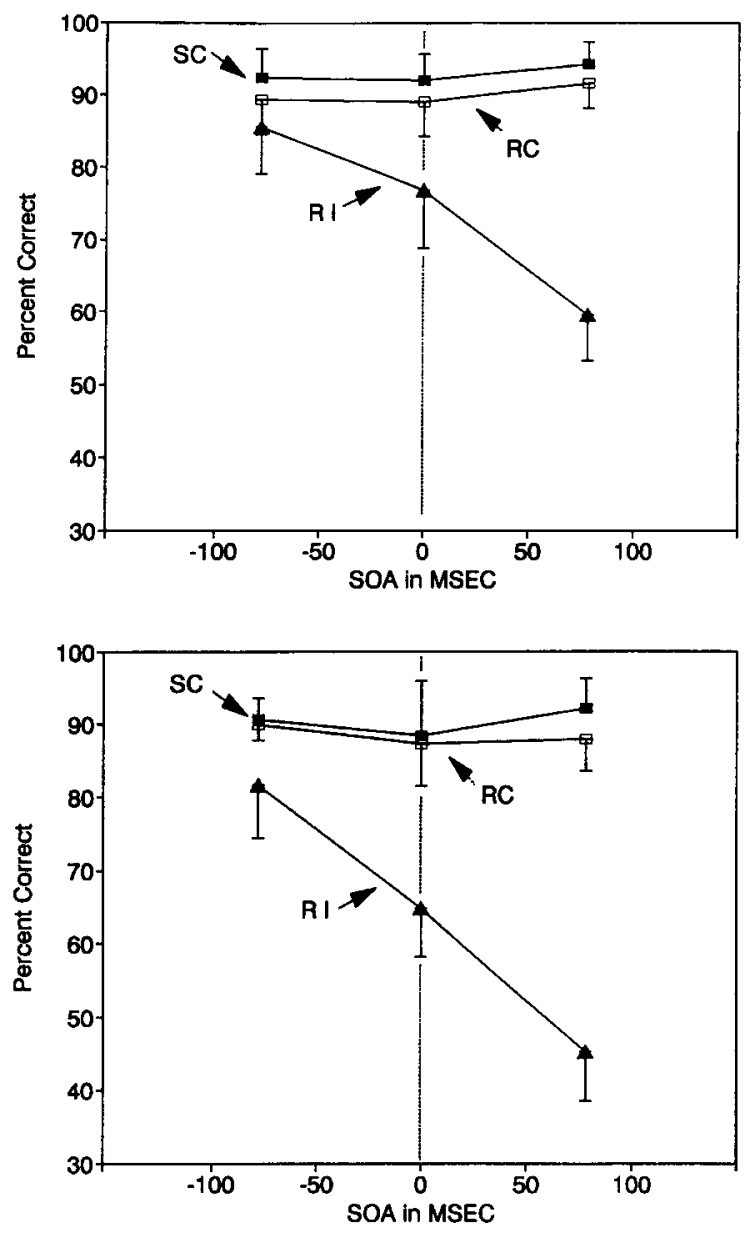

Figure 2. Percent correct identification for the three trial types stimulus compatible (SC), response compatible (RC), and response incompatible (RI)-as a function of SOA. In the upper panel, the target site was the little finger and the nontarget site was the ring finger. In the lower panel, the target site was the ring finger and the nontarget site was the little finger. Error bars represent $1 S E M$.

change in the relative intensity of the target and nontarget did appear to increase the amount of interference (although only leading nontargets were tested). Additional measurements are needed with pairs of patterns that differ in intensity and that cover a range of SOAs.

As suggested above, the results of Experiment 1 are similar to the results of studies conducted under the general rubric of "pattern masking." When two tactile patterns are presented to the same site in rapid succession, subjects have difficulty in identifying a target pattern, whether it is followed by a nontarget (backward-masking paradigm) or preceded by a nontarget (forward-masking paradigm) (Evans \& Craig, 1986). The time course of masking is similar to the time course of the responsecompetition effect; that is, most of the interference occurs within a temporal window of $50-100 \mathrm{msec}$. The similarity of the results from Experiment 1 to the earlier results of temporal-masking studies suggests that similar processes or mechanisms may underlie the reported 
effects. Although one might be tempted to attribute the interference seen in the selective-attention paradigm to what has traditionally been referred to as "masking," we see, with the 4-to-2 paradigm, very little interference on $\mathrm{RC}$ trials when the nontarget is physically different from the target but evokes the same response as the target. We reject a masking explanation for the selective-attention results but consider the alternative: Masking may be due, in part, to the mechanism thought to be responsible for the interference effects seen in the selective-attention paradigm, namely, response competition. Experiment 2 examined this possibility.

\section{EXPERIMENT 2}

In Experiment 2, a temporal-masking paradigm was used in which the target and nontarget stimuli were presented to the same site on the skin. For the most part, previous temporal-masking studies have been conducted with stationary patterns, patterns that were generated at one location on the fingerpad and did not move across the fingerpad. In the present study, the stimulus dimension that distinguishes the four patterns from one another is direction of movement across the fingerpad. In none of the previous studies of masking was direction of movement the relevant stimulus dimension. The first aim of Experiment 2 was to measure the temporal course of masking with the same stimuli that were used in Experiment 1 . The second aim was to examine the role of response competition in a temporal-masking paradigm.

The 4-to-2 paradigm and the same stimuli used in Experiment 1 were used in Experiment 2. If presenting the target and nontarget to the same site produced considerable interference in the early stages of processing (masking), one would expect that when the target and nontarget were physically different, target identification would be poor. In other words, performance on RC and RI trials would be similar to one another and much lower than on SC trials. If both the target and nontarget were processed to the point of evoking competing responses, then, as in Experiment 1, performance on SC and RC trials would be similar and performance on RI trials would be much lower.

Two sets of measurements were obtained. For the first set, the site of stimulation was, as in Experiment 1, the left little finger. For the second set of measurements, the site was switched to the left index finger. The left index finger has been used in previous studies of temporal masking (Craig, 1976, 1982, 1985) and in reading with the Optacon. The second set of measurements was included to test the generality of the findings for other sites of stimulation.

\footnotetext{
Method

Subjects. Eight subjects were tested for the first set of measurements. Seven were right-handed and one was left-handed. One of these subjects had participated in Experiment 1. Four subjects, one of whom had participated in Experiment 1, were tested for the second set of measurements.
}

Apparatus. With the exception that only one tactile array was used, the apparatus was the same as that used in Experiment 1.

Procedure. The procedure was similar to that used in Experiment 1 . The major difference between the two experiments was that both the target and nontarget stimuli were presented to the same site, the pad of the left little finger. Six SOAs were tested, $-500,-100,-52,+52,+100$, and +500 . Trials were blocked by SOA. Each block consisted of 50 trials. Each testing session consisted of seven blocks, including one block in which only the target was presented. The subjects were tested for eight sessions.

In the blocks of trials in which both the target and nontarget were presented, a message remained on the visual display instructing the subjects to respond with the first stimulus (target led the nontarget) or with the second stimulus (target trailed the nontarget). As in Experiment 1, for 4 of the subjects, the mapping of the four stimuli onto the two responses was such that movement to the left or up the finger was categorized as " 1 ," movement to the right or down the finger as "2." For the remaining 4 subjects, the stimulus categories were changed such that left or down was " 1 " and right or up was " 2 ." The stimuli were switched to be sure that there was nothing in a particular grouping of the stimuli that might affect the pattern of results. The other details of the procedure were the same as in Experiment 1.

With the second set of measurements, the site of stimulation was switched to the left index fingerpad. Four SOAs were tested, $-150,-78,+78$, and +150 msec. Each session consisted of five blocks of 50 trials each, and the subjects were tested for seven sessions.

\section{Results and Discussion}

The measurements obtained with the little finger as the site of stimulation were first examined to see if the particular pairing of stimulus direction and responses had had any effect on the results. The mean difference across conditions was less than $6 \%$, and the pattern of results across trial types was the same for the two groups of subjects. The results were combined and are shown in Figure 3. Single-pattern performance was $91 \%$ correct. The results show a greater amount of interference on RI trials when the target and nontarget signaled different responses. Also, as was true in Experiment 1, this effect appears to depend upon the temporal separation between the target and nontarget. A more detailed analysis supported these observations. There was a significant effect of SOA $[F(5,35)=7.38, p<.001]$, a significant effect of trial type $[F(2,14)=23.64, p<.001]$, and a significant interaction between SOA and trial type $[F(10,70)=$ $18.88, p<.001]$.

A comparison of the function shown in Figure 3 with the function shown in Figure 1 reveals some similarities and some differences between presentation of a nontarget to the same site as the target and presentation of a nontarget to a different site. The first and perhaps most important similarity is that the performance seen on RC trials is similar to that seen on SC trials and considerably better than that obtained on RI trials. Where the differences were greatest in the selective-attention task, that is, the four briefest SOAs, (Figure 1), performance averaged $82 \%$ correct on SC trials, $78 \%$ correct on RC trials, and $56 \%$ correct on RI trials. These results support one of the conclusions of Experiment 1, namely that considerable interference results from trials in which the 


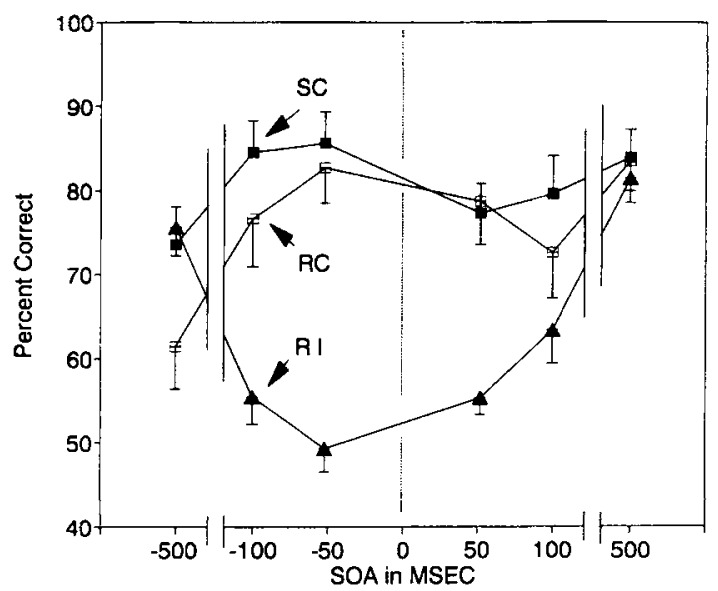

Figure 3. Percent correct identification for the three trial typesstimulus compatible (SC), response compatible (RC), and response incompatible (RI) - as a function of SOA. Both the target and nontarget were presented to the little finger. Error bars represent $1 S E M$.

nontarget is associated with a response different from that of the target (RI trials). Much less interference is seen with a nontarget that is physically different from the target but associated with the same response ( $\mathrm{RC}$ trials). These results suggest that a considerable amount of the interference that occurs between stimuli presented to the same site may be due more to response competition and less to interference at early stages of processing, such as masking.

As expected, there is considerably more overall interference when the target and nontarget are presented to the same site than when they are presented to different sites. In a selective-attention paradigm, even with no temporal separation between the target and nontarget, performance rarely falls to chance levels. Subjects are able to use location information to keep the target and nontarget separate, although with less than $100 \%$ success. When spatial cues are removed and the target and nontarget are presented to the same site, performance declines.

Two aspects of the functions shown in Figure 3 are unexpected. First, performance on the $\mathrm{SC}$ and $\mathrm{RC}$ trials declines as the SOA increases from -52 to -100 to $-500 \mathrm{msec}$. This finding is unexpected, because in no previous study of masking of which we are aware did performance decline with increasing temporal separation. Also, performance declines on both RC and SC trials, trials on which the nontarget is associated with the same response as the target. In one earlier study, nontargets (maskers) identical to the target were tested, and these produced virtually no interference (Craig \& Evans, 1987). In this earlier study, the target and maskers were stationary patterns. At present, we can offer only the speculation that the interference seen at longer SOAs may be related to the fact that the stimuli in the present study are moving patterns and that identification is based on direction of motion. Additional measurements with both stationary and moving stimuli will be needed to determine more precisely the nature of the interference effect at long SOAs.

The second unexpected finding is that a trailing nontarget does not produce more interference than a leading nontarget. Previous studies had rather consistently found that backward maskers produce more interference in a pattern-identification task than did forward maskers (Craig, 1976, 1982, 1985; Evans, 1987; Evans \& Craig, 1986). This result was found both with stationary patterns and with patterns scanned across the tactile display. A Tukey $H S D$ test revealed no significant differences between the percentage correct at -52 and $+52 \mathrm{msec}(p>$ .05 ), the briefest SOA, where the largest decrement for backward maskers has been reported. In light of the results from Experiment 1 and previous temporal-masking results, the question is why we did not see more backward than forward interference. One possible answer is that when the target and nontarget were moved to the same site (a major difference between Experiments 1 and 2), the possibility for generating apparent motion between the two stimuli increased. For stimulus durations of $50 \mathrm{msec}$, best apparent motion is generated with SOAs between 75 and $100 \mathrm{msec}$ (Sherrick \& Rogers, 1966) - that is, within the range of the SOAs tested in the present study. If apparent motion were created between the target and nontarget stimulus, then subjects would receive three patterns of motion on trials at brief SOAs, one pattern of movement from the target, one from the nontarget, and one generated from the interaction of the target and nontarget. On SC and RC trials, two of three patterns of motion are signaling the same, correct response.

If subjects are basing their decisions on the perceived direction of motion, then apparent motion between the target and nontarget might well interfere in target identification. Presumably the strength of the apparent motion generated would be independent of the temporal order of the target and nontarget and thus might tend to produce equal amounts of forward and backward interference. In other masking studies in which subjects' responses are based on pattern shape, apparent motion might be expected to have little effect on performance. The greater backward interference seen in these earlier studies might be the result of the stronger representation of the trailing pattern (Evans \& Craig, 1986).

The index finger results provide some additional forward/backward interference data. These results are shown in Figure 4. Single-pattern performance at this site was $96 \%$ correct. The main reason for including these measurements was to see if the results with the index finger as target site, the site used in a number of earlier studies of pattern perception and in reading with the Optacon, would be comparable to those obtained on the little finger. A two-way, repeated measures ANOVA with the index finger as the target site showed no effect of SOA $[F(3,9)=1.70, p>.05]$, a significant effect of trial type $[F(2,6)=18.39, p<.01]$, and no significant interaction between SOA and trial type $[F(6,18)=0.57$, $p>.05]$. The results are similar to those obtained on the 
little finger in that there is considerable interference only on those trials (RI trials) when the response is different from that of the target. Modest interference is seen when the nontarget is physically different from the target but associated with the same response ( $\mathrm{RC}$ trials). Presenting the same stimulus ( $\mathrm{SC}$ trials) also results in modest amounts of interference. Perhaps because of the relatively brief range of SOAs tested, these results show no effect of SOA on performance.

The fact that there is no significant effect of SOA and no interaction between trial types and SOA indicates that, as before, a backward nontarget does not produce more interference than a forward nontarget. Taken together, the results presented in Figures 3 and 4 are inconclusive with regard to the relative effectiveness of forward versus backward nontargets. Because previous masking studies with stationary stimuli consistently showed more backward than forward masking, it may be reasonable to assume that not finding such an effect in the present study might be due to the nature of the moving stimuli that were used. Additional measurements with stationary stimuli using the 4-to-2 paradigm will be needed to determine the extent to which the paradigm itself might affect the temporal course of interference.

Because of the unexpected drop in performance at longer forward SOAs with patterns presented to the little finger (Figure 3), we tested a longer SOA with patterns presented to the pad of the index finger. Four subjects were tested with two SOAs, one at $-52 \mathrm{msec}$ and one at $-500 \mathrm{msec}$. The results were similar to those shown in Figure 3. For RI trials, performance was better at $-500 \mathrm{msec}$ than at $-52 \mathrm{msec}$. For SC and RC trials, performance was worse at the longer SOA. Performance on SC and RI trials was nearly identical at $-500 \mathrm{msec}$, with performance lower on RC trials. The surprising drop in performance with these stimuli at longer SOAs on SC and RC trials appears not to be limited to patterns presented to the little finger.

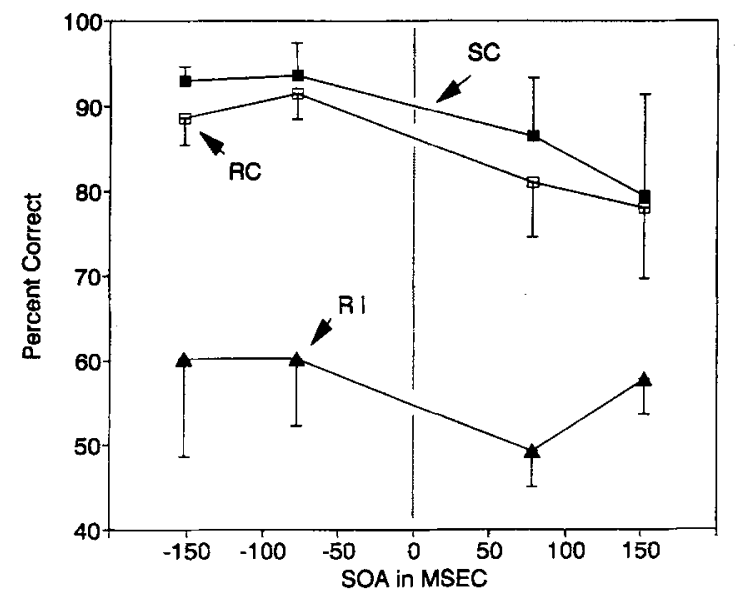

Figure 4. Percent correct identification for the three trial typesstimulus compatible (SC), response compatible ( $\mathrm{RC}$ ), and response incompatible (RI) - as a function of SOA. Both the target and nontarget were presented to the index finger. Error bars represent $1 S E M$.
The results of Experiment 2 indicate that response competition is a major factor in masking. A responsecompetition model implies that both the target and nontarget are fully processed and available as responses. In previous work with a selective-attention paradigm similar to that used in Experiment 1 (Evans \& Craig, 1992), the data have supported the view that both the target and the nontarget are available as responses. In switching to a masking paradigm, it is appropriate to raise the issue again and to ask whether the target and nontarget are both fully processed or whether the nontarget interferes with the target by replacing it. Perhaps at the time the subject responds, only one stimulus representation may be available, the nontarget. If the nontarget is identical to the target ( $\mathrm{SC}$ trials) or if it evokes the same response (RC trials), the subject responds correctly. If the nontarget evokes a different response (RI trials), the subject responds incorrectly. With only two responses possible, as in the present study, the percentage of correct responses does not differentiate between the two views, response competition and replacement; however, the reaction time data in Experiment 2 help to sort out the two views. If the masker replaces the target and subjects have only one stimulus representation available on each trial, then reaction times for the three types of trials should be similar.

For the reaction time data from the little finger site, the ANOVA showed a significant effect of $\operatorname{SOA}[F(5,35)=$ $9.96, p<.0001]$, a significant effect of trial type $[F(2,14)=$ $14.78, p<.001]$, and a significant interaction between SOA and trial type $[F(10,70)=4.12, p<.001]$. The average reaction times were $574 \mathrm{msec}$ on SC trials, $589 \mathrm{msec}$ on RC trials, and $610 \mathrm{msec}$ on RI trials. For the reaction time data for the index finger, the ANOVA showed a significant effect of SOA $[F(3,9)=7.64, p<.01]$, a significant effect of trial type $[F(2,6)=6.58, p<.05]$, and no significant interaction. The pattern of reaction times across the three trial types was similar to that for the little finger; the average reaction times were $531 \mathrm{msec}$ for SC trials, $564 \mathrm{msec}$ for RC trials, and $648 \mathrm{msec}$ for RI trials. The reaction times are computed on correct trials only.

The fact that reaction times differ by trial type for both sets of data indicates that subjects are taking significantly longer to respond correctly on those trials when the nontarget differs from the target. The reaction time results indicate that representations of both the target and nontarget are available to the subjects at the time they respond. As in Experiment 1, the longer reaction times on RI trials presumably result from subjects' taking time to choose between competing responses.

It may not be surprising that when selective attention fails, the pattern of results resembles those obtained when a target and nontarget are presented to the same location. Selective attention fails when a spatial cue, location, is ineffective in separating the target and nontarget stimuli. Presenting the target and nontarget to the same location produces a similar effect, that is, location becomes an ineffective cue for separating the target and nontarget. What may be surprising is that response com- 
petition, the mechanism thought to be responsible for interference in selective attention, may account for a significant portion of what has been termed "masking."

By "masking" we mean that the nontarget (masker) interferes with the perception of the target by altering the representation of the target at a relatively early stage of processing. Indeed, several studies have found evidence that the target is altered by such factors as temporal integration (Evans, 1987; Evans \& Craig, 1986), spatial distortion (Craig, 1989), and so forth. The present results suggest that these factors are insufficient to account for the total amount of interference produced by nontargets. If response competition can be shown to operate more generally, it indicates that a major limitation in the processing of sequences of spatial patterns is the perception of the temporal order of the patterns (Craig \& Xu, 1990). In other words, subjects may have two relatively clear representations, a target and a nontarget, and, if the sole basis for distinguishing the two is temporal order, errors at brief separations are likely.

How pervasive might response competition be in temporal-masking studies? What the present study has shown is that when highly identifiable, moving patterns are presented to the same location, much less interference is observed if the two stimuli differ only physically from one another than if the two stimuli differ from one another and are associated with different responses. These results would lead one to predict that the amount of interference observed in a masking paradigm would be directly related to the degree that the nontargets (maskers) elicited competing responses. There is some qualitative support for this prediction. In an earlier study, in which the target patterns were letters, an energy masker, created by simultaneously turning on all pins in the tactile array, produced less interference than did pattern maskers made up of elements of letters (Craig, 1982). Presumably the pattern maskers felt more like letters than did the energy masker, and as such were more likely to evoke a response that competed with the target response.

The results of Experiments 1 and 2 suggest that a major limitation in processing complex tactile stimuli is the inability to organize the patterns temporally and spatially. The separate patterns arriving at either the same or different fingerpads may be relatively clearly registered and suffer little degradation at early stages of processing. The difficulty arises in knowing at what location or in what temporal order the patterns were received (Craig $\& \mathrm{Xu}, 1990)$. The need to provide some ordering for patterns received over space and time may be particularly critical in haptic exploration of objects. Successful identification of an object may require some overall, topdown processing schema to keep the various features or- dered. Such speculations suggest the importance of the stereotypical exploratory procedures that are used in the haptic identification of objects (Klatzky \& Lederman, 1993; Klatzky, Lederman, \& Reed, 1987; Lederman \& Klatzky, 1987).

\section{REFERENCES}

CRAIG, J. C. (1976). Vibrotactile letter recognition: The effects of a masking stimulus. Perception \& Psychophysics, 20, 317-326.

Craig, J. C. (1980). Modes of vibrotactile pattern generation. Journal of Experimental Psychology: Human Perception \& Performance, 6 , 151-166.

Craig, J. C. (1982). Vibrotactile masking: A comparison of energy and pattern maskers. Perception \& Psychophysics, 31, 523-529.

Craig, J. C. (1985). Tactile pattern perception and its perturbations. Journal of the Acoustical Society of America, 77, 238-246.

Craig, J. C. (1989). Interference in localizing tactile stimuli. Perception \& Psychophysics, 45, 343-355.

Craig, J. C., \& Evans, P. M. (1987). Vibrotactile masking and the persistence of tactual features. Perception \& Psychophysics, 42, 309317.

Craig, J. C., \& Xu, B. (1990). Temporal order and tactile patterns. Perception \& Psychophysics, 47, 22-34.

Eriksen, C. W., O'Hara, W. P., \& ERIKSEn, B. (1982). Response competition effects in same-different judgments. Perception \& Psychophysics, 32, 261-270.

ERIKSEN, C. W., \& SCHULTZ, D. W. (1979). Information processing in visual search: A continuous flow conception and experimental results. Perception \& Psychophysics, 25, 249-263.

Evans, P. M. (1987). Vibrotactile masking: Temporal integration, persistence, and strengths of representation. Perception \& Psychophysics, 42, 515-525.

Evans, P. M., \& CRAIG, J. C. (1986). Temporal integration and vibrotactile backward masking. Journal of Experimental Psychology: Human Perception \& Performance, 12, 160-168.

Evans, P. M., \& Craig, J. C. (1991). Tactile attention and the perception of moving tactile stimuli. Perception \& Psychophysics, 49, 355364.

Evans, P. M., \& Craig, J. C. (1992). Response competition: A major source of interference in a tactile identification task. Perception \& Psychophysics, 51, 199-206.

Evans, P. M., Craig, J. C., \& Rinker, M. A. (1992). Perceptual processing of adjacent and nonadjacent tactile nontargets. Perception \& Psychophysics, 52, 571-581.

KLATZKY, R. L., \& LeDERMAN, S. J. (1993). Toward a computational model of constraint-driven exploration and haptic object identification. Perception, 22, 597-621.

KLATZKy, R. L., Lederman, S. J., \& ReEd, C. (1987). There's more to touch than meets the eye. Journal of Experimental Psychology: General, 116, 356-369.

LEDERMAN, S. J., \& KLATZKY, R. L. (1987). Hand movements: A window into haptic object recognition. Cognitive Psychology, 19, 342368.

Rinker, M. A., \& CraIG, J. C. (1994). The effects of spatial orientation on the perception of moving tactile stimuli. Perception \& Psychophysics, 56, 356-362.

SHERrick, C. E., \& Rogers, R. (1966). Apparent haptic movement. Perception \& Psychophysics, 1, 175-180.

(Manuscript received June 27, 1994; revision accepted for publication December 11, 1994.) 